\title{
The Potential Application of EBSD to Serial Number Restoration
}

\author{
Carl Necker, Robert Forsyth
}

Los Alamos National Laboratory, Material Science and Technology: Metallurgy, MS: G770, Los Alamos, NM 87545

The restoration of defaced serial numbers from weapons and other items plays a key role in forensic analysis. The restoration process typically requires polishing and etching with a specific acid to attack the residual deformation caused by the initial impression process. This etching process may provide an optical 'ghost' image of the original impression. Figure 1 shows a serial number after the initial grinding process using 240 grit silicon carbide paper. The right image in Figure 1 was taken after the grinding operation completely removed the '193' (CY and '7' were embossed more deeply and were not completely removed). Then the surface was polished and etched using Fry's etch to reveal the ghost image. The success rate is typically $60 \%-80 \%$ on stamped impressions with much lower success rates for engraved or laser etched impressions. An alternative means of restoring the serial number may be through the use of an electron microscopy technique, electron backscatter diffraction. The process requires polishing and light etching or electropolishing for optimal results. The electron beam is moved through a surface array of points where the crystallographic orientation is captured at each point. Figure 2 shows a typical electron backscatter pattern. The colored bands in the overlay represent the best-fit calculated crystal orientation for that pattern. The collective crystallographic information from all the patterns can be used to reconstruct the microstructure and then assess the microstructure and texture using image analysis techniques. Figure 3 shows a variety of maps from a scan covering the absent ' 9 '. Please note that the ' 9 ' is inverted in these maps due to the sample orientation inside the electron microscope. The left-most map is colored according to the crystal direction of each data point. The ' 9 ' can be faintly seen. The next map is an image quality map, a measure of how clearly the software recognized the presence of each pattern. The image quality is a function of how disordered the local crystal structure is. The greater the degree of local deformation, the worse the image quality is. The ' 9 ' is clearly seen. The next map is the confidence index map. It defines how clearly the software can calculate the orientation of the pattern. Again, the ' 9 ' is clearly seen. The right-most map is a kernel average misorientation map. The color-coding represents the degree of misorientation between neighboring data points. The greater the deformation, as would be found under a stamped impression, the greater the local misorientations are. This scan, captured at $5 \mu \mathrm{m}$ resolution, took about 2 hours to produce. Additional test scans at $10 \mu \mathrm{m}$ and $20 \mu \mathrm{m}$ provided equally clear results with the $20 \mu \mathrm{m}$ resolution scan taking only about 5 minutes to cover the ' 9 '.

In this study, we explore the sample processing requirements and assess initial EBSD scan results on stamped low carbon steel to determine if EBSD can recover serial numbers. Initial results are promising, showing that individual numbers are clearly re-established after the serial number has been ground away. Future studies will determine how far below the bottom of the visible impression EBSD can re-establish serial number evidence in a variety of metallic materials. 

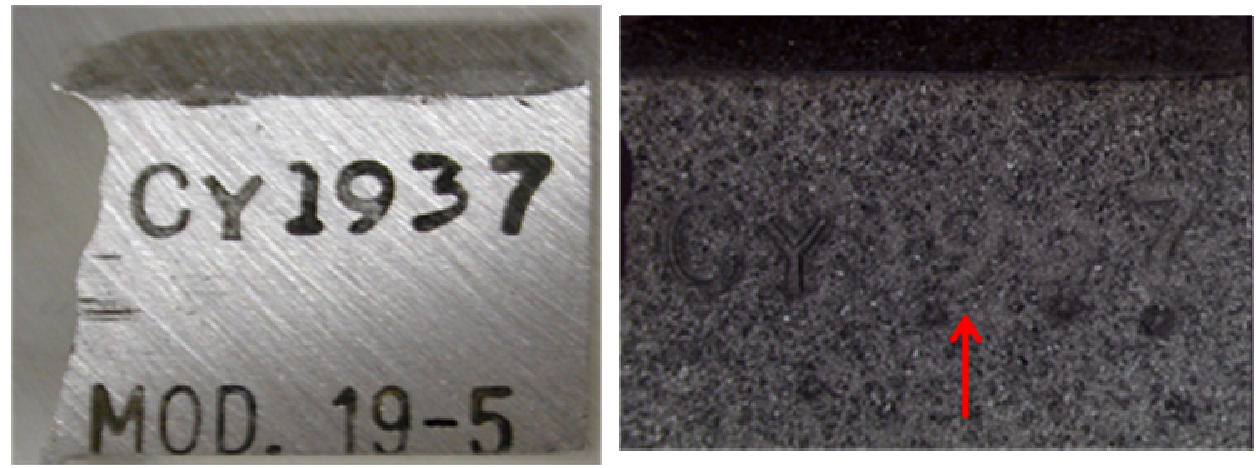

Figure 1. Left - serial number after initial grinding step. Right - serial number following after ' 9 ' has been ground away, shown in etch relief (arrow).

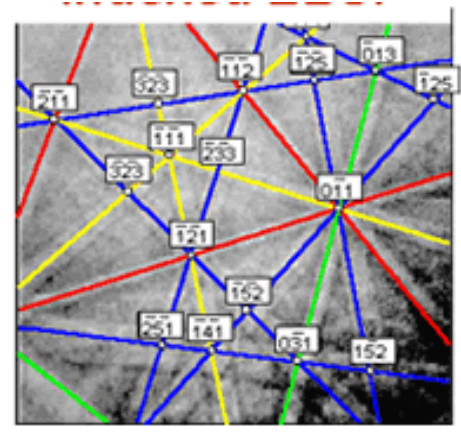

Figure 2. Typical EBSD pattern with calculated crystal orientation overlay.
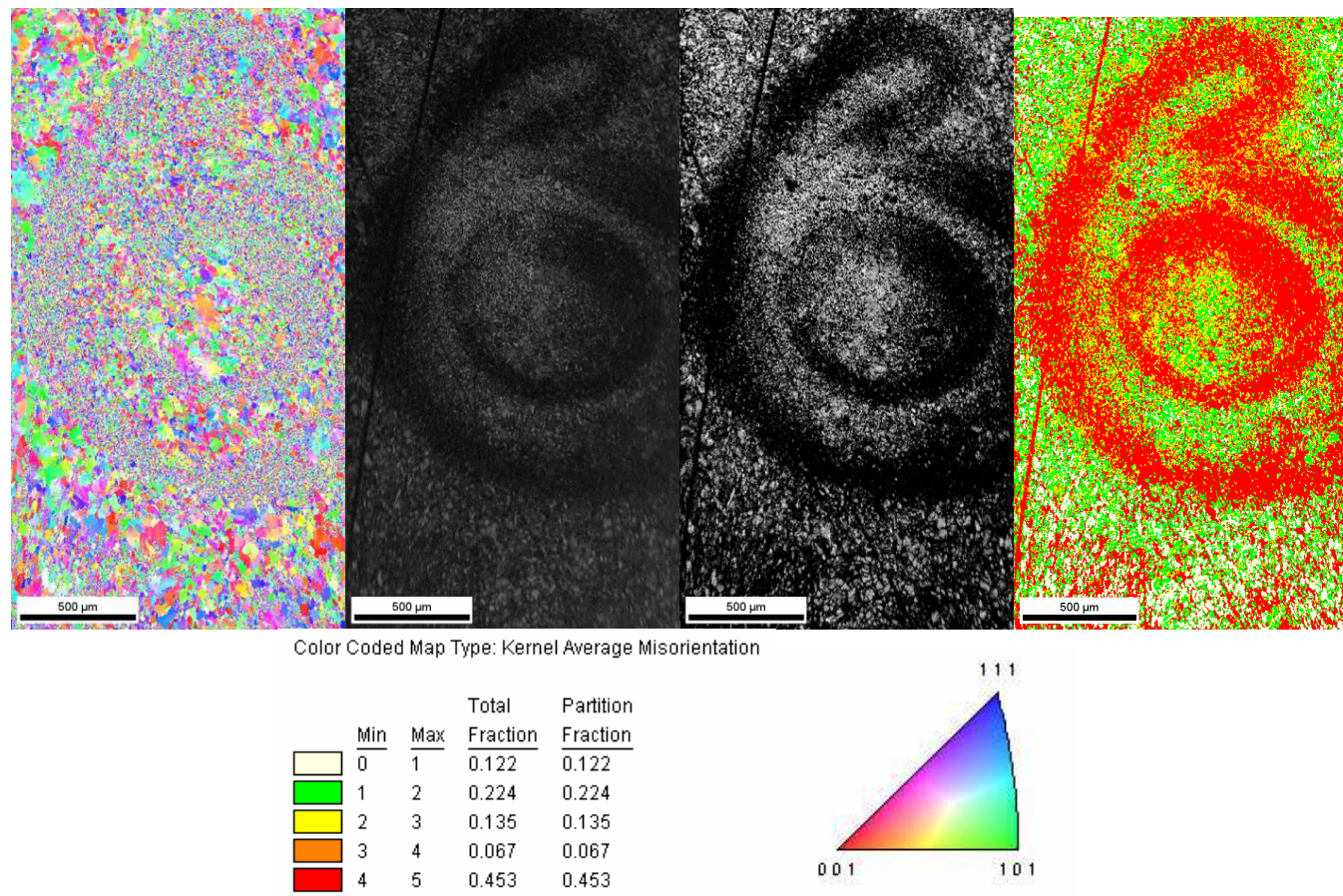

Figure 3. Left to Right - crystal direction map constructed from $5 \mu \mathrm{m}$ step size scan over the ' 9 '; image quality map; confidence index map; kernel average misorientation map. 